\title{
Aquiferspeicher in Deutschland
}

\author{
Paul Fleuchaus $^{1} \cdot$ Simon Schüppler ${ }^{2} \cdot$ Ruben Stemmle $^{1} \cdot$ Kathrin Menberg $^{1} \cdot$ Philipp Blum $^{1}$
}

Eingegangen: 26. Juli 2020 / Überarbeitet: 8. Dezember 2020 / Angenommen: 13. Januar 2021 / Online publiziert: 19. Februar 2021

(c) Der/die Autor(en) 2021

\section{Zusammenfassung}

Der saisonale Versatz von Angebot und Nachfrage im Wärmesektor kann über Speicherlösungen ausgeglichen werden. Für die jahreszeitliche Speicherung von Wärme und Kälte sind Aquiferspeicher (ATES) als vielversprechende Lösung vermehrt in den Fokus gerückt. Mit derzeit jeweils nur einem betriebenen Niedrigtemperatur- (NT) und Hochtemperaturspeicher (HT) fristet die Technologie in Deutschland allerdings noch immer ein Nischendasein. Diese Studie liefert einen Überblick über die aktuelle Entwicklung der Aquiferspeicherung in Deutschland und diskutiert Stärken und Schwächen sowie Chancen und Risiken. Trotz eines großen Nutzungspotenzials wird der Markteinstieg in Deutschland durch fehlende Anreizprogramme, mangelnde Kenntnisse sowie nicht vorhandene Pilotanlagen erschwert. Die Speichertemperaturen von HT-ATES $\left(>50^{\circ} \mathrm{C}\right)$ erhöhen dessen Nutzungsmöglichkeiten, haben aber verstärkte technische und legislative Risiken zur Folge. Eine kommerzielle ATES-Nutzung in Deutschland ist daher nur möglich durch die Anpassung genehmigungsrechtlicher Anforderungen, die Schaffung von Fördermaßnahmen, die Umsetzung von Demonstrationsanlagen und die Darlegung von deren wirtschaftlichen und ökologischen Vorteilen.

Schlüsselwörter Geothermie · Wärmespeicher $\cdot$ Saisonale Speicherung $\cdot$ Untergrundspeicher

\section{Aquifer Thermal Energy Storage (ATES) in Germany}

\begin{abstract}
The time offset between supply and demand in the energy sector can be equalized with seasonal energy storage (at relatively warm or cold temperatures). For the latter, aquifer thermal energy storage (ATES) is considered a promising solution. However, with only a single low-temperature (LT) and another high-temperatures (HT) storage system currently in operation, ATES is little known and not well established in Germany. This study provides an overview of the current ATES development in Germany and discusses strengths and weaknesses, as well as opportunities and risks. Despite a great potential, the market entry of ATES in Germany is impeded by the absence of incentive plans, lack of knowledge and non-existent pilot systems. Storage temperatures of HT-ATES $\left(>50^{\circ} \mathrm{C}\right)$ have increased its potential for use, yet result in increased technical and legislative risks. Thus, the commercial application of ATES in Germany is only feasible through the adjustment of legislative requirements, the implementation of funding programs and installation of demonstration plants proving the economic and ecological benefits.
\end{abstract}

Keywords Geothermal energy $\cdot$ Aquifer thermal energy storage $\cdot$ Seasonal energy storage

Paul Fleuchaus

paul.fleuchaus@kit.edu

1 Institut für Angewandte Geowissenschaften, Karlsruher Institut für Technologie (KIT), Adenauerring

20b, 76131 Karlsruhe, Deutschland

2 EIfER - Europäisches Institut für Energieforschung EDF-KIT, Emmy-Noether-Str. 11, 76131 Karlsruhe, Deutschland

\section{Einleitung}

Die zu erwartenden Folgen des Klimawandels erfordern eine drastische Reduzierung der globalen Treibhausgasemissionen. Während in Deutschland der Anteil erneuerbarer Energien im Stromsektor in den vergangenen Jahren stetig gestiegen ist, stagniert dieser im Wärme- und Kältesektor im letzten Jahrzehnt bei rund 14\% (176,5 TWh) (Umweltbundesamt 2019). Obwohl die Wärme- und Kälteversor- 
gung von Gebäuden mit 1260 TWh rund die Hälfte des Endenergieverbrauches ausmacht (Umweltbundesamt 2020), herrscht aufgrund des jahreszeitlichen Versatzes zwischen Wärmeangebot und -nachfrage in Deutschland weniger ein Energie- als ein Speicherproblem. Folglich ist in den letzten Jahren das Interesse an thermischen Speichern stark gewachsen. Die saisonale Speicherung von Wärme und Kälte in Grundwasserkörpern, auch Aquiferspeicherung (ATES) genannt, zeichnet sich im Vergleich zu anderen Speichertechnologien durch geringe Speicherkosten (Mangold et al. 2012; Holstenkamp et al. 2017; Schüppler et al. 2019) und hohe Speicherkapazitäten (Kranz et al. 2008; Schmidt et al. 2004; Andersson 2007) aus, und ist in den vergangenen Jahren insbesondere in der Forschung verstärkt in den Fokus gerückt (Bloemendal et al. 2015; Bloemendal 2018; Fleuchaus et al. 2018; Fleuchaus 2020).

Mit einer Entwicklungsgeschichte von über 50 Jahren haben sich verschiedene Konzepte und Nutzungsformen der Aquiferspeicherung entwickelt (Fleuchaus et al. 2018; Dickinson et al. 2009). Die wichtigste Unterscheidung erfolgt anhand der Speichertemperatur in Niedrigtemperatur (NT)- und Hochtemperatur (HT)-Systeme. NT-ATES lassen sich über eine maximale Speichertemperatur von $25^{\circ} \mathrm{C}$ definieren (vereinzelt auch bis $40^{\circ} \mathrm{C}$ ) und werden meist für die Beheizung und Klimatisierung großer öffentlicher oder kommerziell genutzter Gebäude in Kombination mit einer Wärmepumpe eingesetzt (Bloemendal 2018; Fleuchaus et al. 2020b; Sommer 2015). Als thermische Energiequelle dient häufig die Abwärme/Kälte des versorgten Gebäudes selbst (Bakema et al. 1995; Snijders 2000). HT-ATES zeichnen sich dagegen durch Speichertemperaturen von mindestens $50^{\circ} \mathrm{C}$ aus (Fleuchaus 2020; Sanner et al. 2003). Aufgrund der hohen Speichertemperaturen ist die Wärmequelle (industrielle Abwärme, erneuerbare Energien) meist unabhängig und getrennt vom Wärmeabnehmer (Fernwärmenetze, industrielle Anwendungen, Gebäudekomplexe). Sowohl NT- als auch HT-Systeme bestehen in ihrer Grundform aus einem oder mehreren Brunnenpaaren (Entnahmeund Eingabebrunnen) (Dickinson et al. 2009). Die Einspeicherung und Wiedergewinnung der Wärme und Kälte erfolgt durch eine saisonale Umkehrung der Pumprichtung (Abb. 1). Dadurch lassen sich Aquiferspeicher eindeutig von klassischen (direkten) Nutzungsformen der oberflächennahen und tiefen Geothermie abgrenzen (Fleuchaus 2020).

Weltweit befinden sich derzeit rund 3000 Aquiferspeicher in Betrieb (Fleuchaus et al. 2018). Trotz eines sehr großen Nutzungspotenzials (Bloemendal et al. 2015; Lu et al. 2019; Kunkel et al. 2019; Stemmle 2020) fristet die Technologie in Deutschland mit aktuell nur zwei betriebenen Systemen noch immer ein Nischendasein (Holstenkamp et al. 2017; Fleuchaus 2020). Ziel dieser Studie ist es deshalb, einen umfassenden Überblick über die aktuelle Aquiferspeicherentwicklung in Deutschland zu geben. Da-

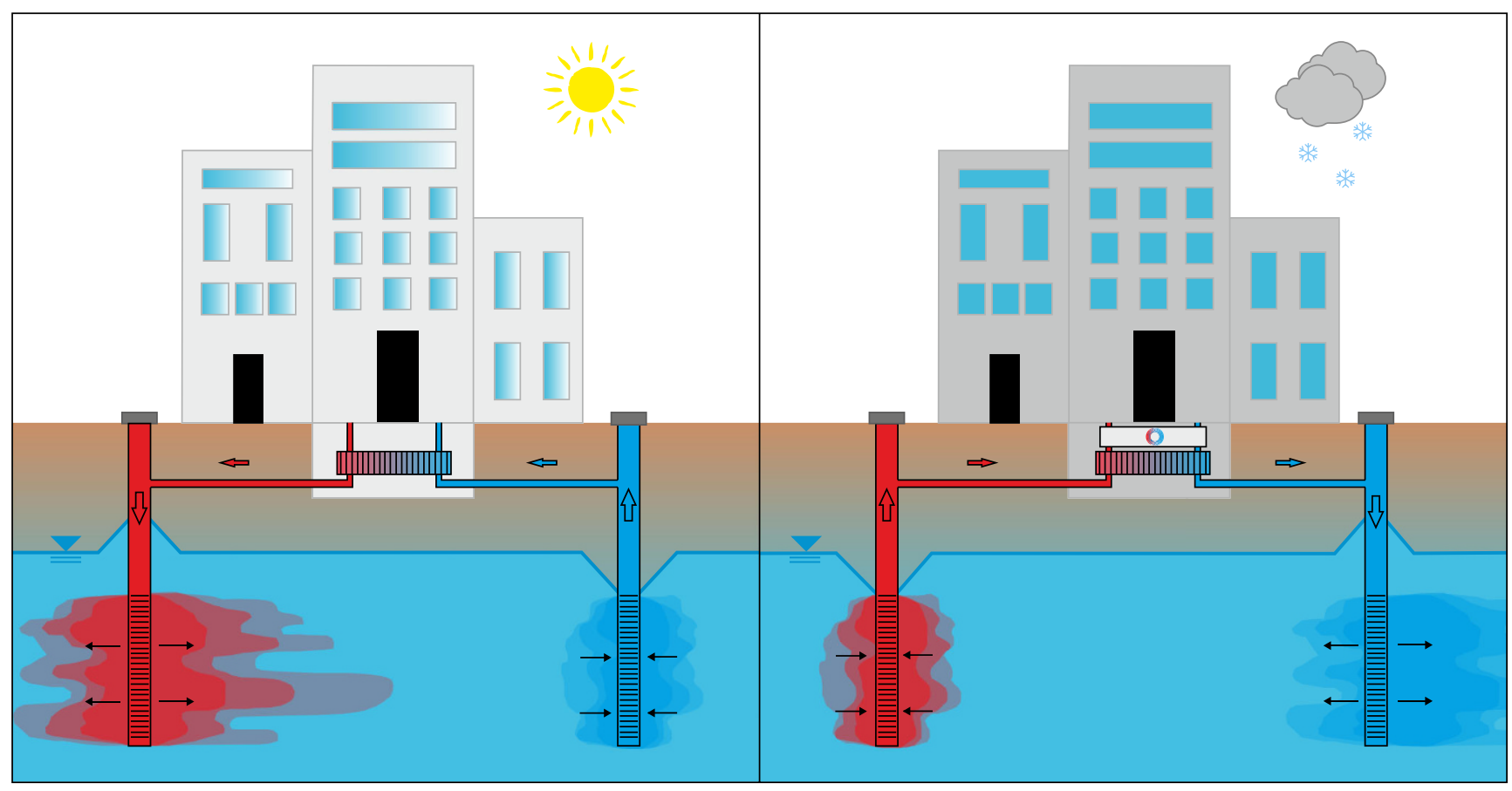

Abb. 1 Grundprinzip eines Niedrigtemperatur-Aquiferspeichers (NT-ATES). Rechts ist der Winterbetrieb zum Heizen und links der Sommerbetrieb zum Kühlen der Gebäude illustriert (Fleuchaus 2020)

Fig. 1 Basic principle of low-temperature aquifer thermal energy storage (NT-ATES). The right hand side shows winter operation for heating, the left hand side illustrates summer operation for cooling of the buildings (Fleuchaus 2020) 
rauf aufbauend sollen anhand einer SWOT-Analyse Stärken, Schwächen, Chancen und Risiken der Technologie in Deutschland analysiert und notwendige Maßnahmen für eine großflächige Nutzung identifiziert werden.

\section{Aquiferspeicher in Deutschland}

Erste Aquiferspeicherversuche wurden in Deutschland in den 1970er und 1980er Jahren in Krefeld (Mercer et al. 1981; Silvermann 1980; Tsang 1980; Werner und Kley 1977) und an der Universität Stuttgart (Adinolfl und Ruck 1992; Adinolfl et al. 1994) durchgeführt. Als Reaktion auf die erste große Ölkrise waren die Versuche Teil des „Energy Conservation through Energy Storage“ (ECES) der International Energy Agency (IEA) und zielten auf die Entwicklung innovativer Speichertechnologien ab (Sanner 2001). In den folgenden Jahren konnte sich die Technologie allerdings nicht am Energiemarkt durchsetzen. Geochemische Wechselwirkungen aufgrund hoher Speichertemperaturen führten bei internationalen Projekten zu erheblichen tech-

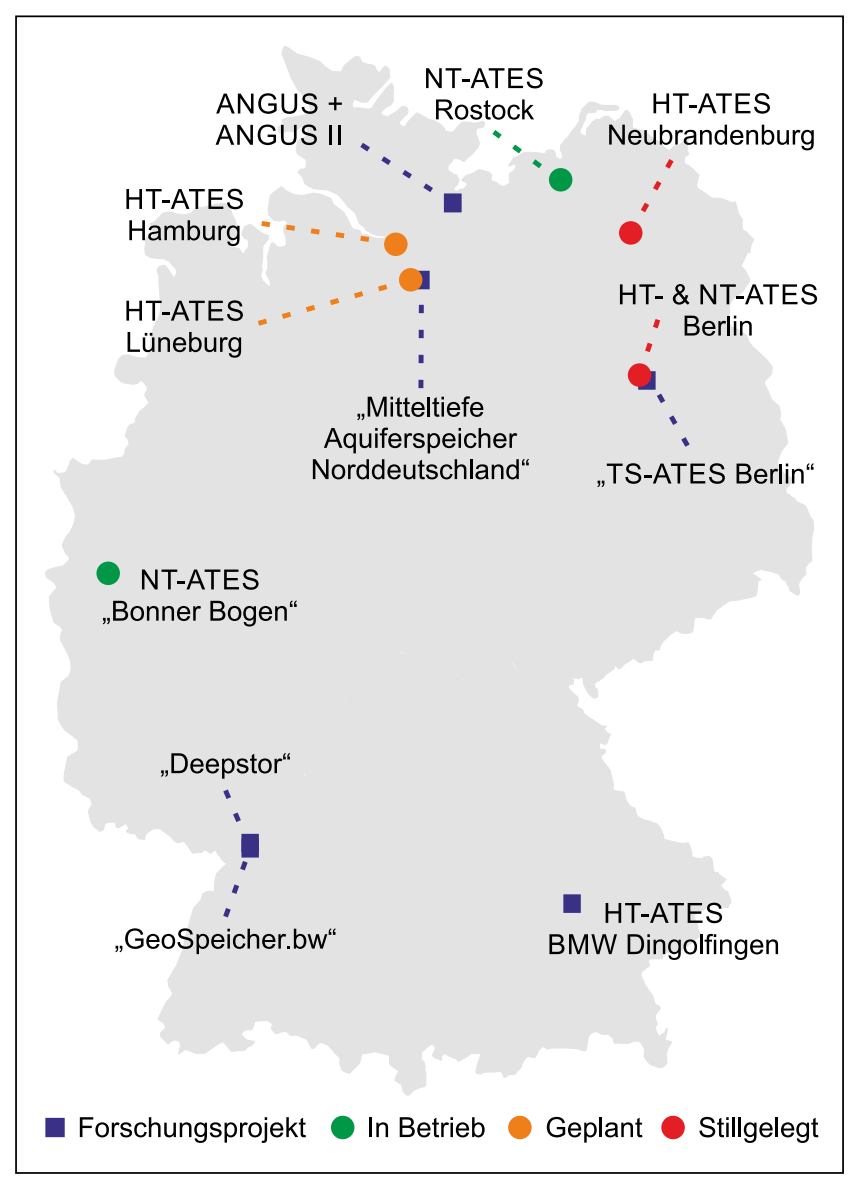

Abb. 2 Übersicht stillgelegter, in Betrieb und in Planung befindlicher Aquiferspeicher in Deutschland

Fig. 2 Spatial distribution of abandoned, operating and planned ATES projects in Germany

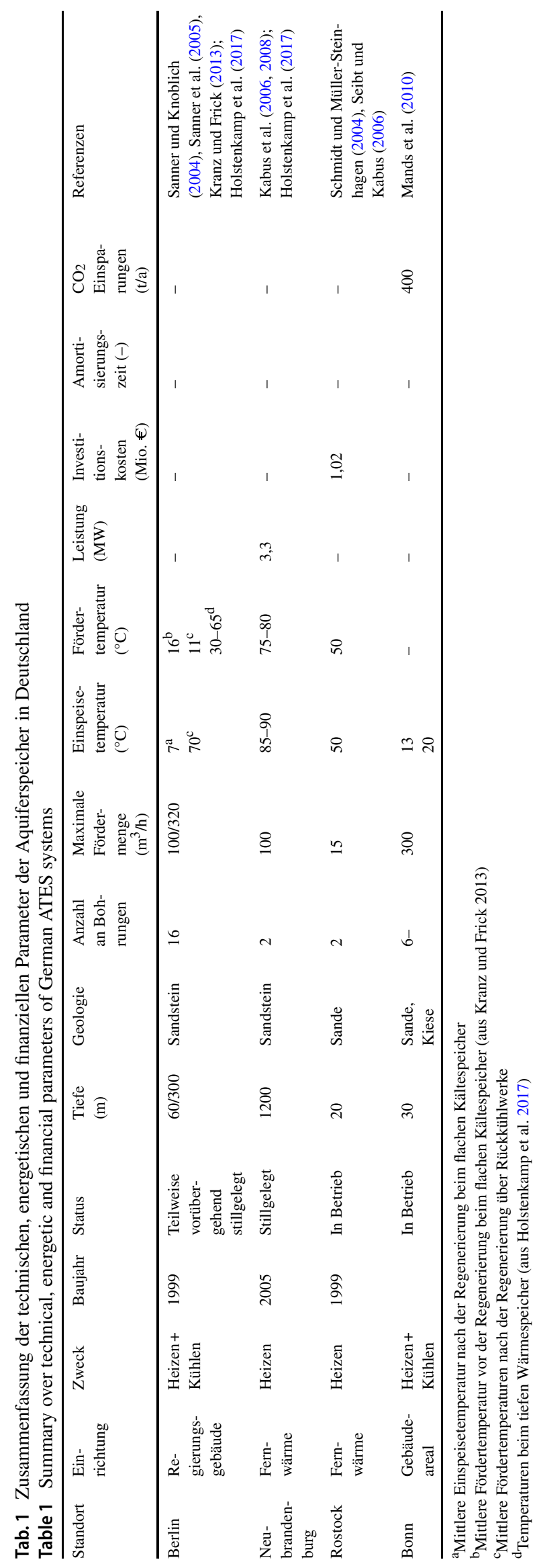


nischen Problemen (Jenne et al. 1992). Stark sinkende Ölund Gaspreise führten zudem zu einer Fokussierung auf die direkte, tiefengeothermische Nutzung zur Stromproduktion. Ein umfassender Überblick über die historische und technische Entwicklung der Aquiferspeicherung ist in Fleuchaus et al. (2018) zu finden. Nachdem um die Jahrtausendwende drei HT- und ein NT-Projekt erfolgreich umgesetzt und über 10 Jahre nachhaltig betrieben wurden, kam es in den letzten Jahren aufgrund neuer Förderprogramme zur Initiierung weiterer Forschungsprojekte. Abb. 2 zeigt die geographische Lage vergangener und aktueller ATES-Projekte in Deutschland nach aktuellem Kenntnisstand der Autoren. Technische, energetische und finanzielle Parameter sind zudem in Tab. 1 zusammengefasst. Im Folgenden werden die wichtigsten Schlüsselinformationen der einzelnen Projekte näher beschrieben.

\section{Berlin}

Die Energieversorgung der Parlamentsbauten am Spreebogen wurde Mitte der 1990er Jahre entworfen und erfolgt überwiegend dezentral über regenerative Primärenergie. Im Rahmen der Neukonzeptionierung wurden im Jahr 1999 zwei Aquiferspeicher für die Wärme- und Kälteversorgung des Reichstaggebäudes (Abb. 3a) sowie der benachbarten Regierungsbauten in Betrieb genommen (Sanner et al. 2005). Für die Kälteversorgung im Sommer erschließen 14 Brunnen einen Aquifer in $60 \mathrm{~m}$ Tiefe. Die Entnahmetemperaturen liegen zwischen 10 und $11^{\circ} \mathrm{C}$ und ermöglichen eine freie Kühlung. Die Regeneration des Speichers erfolgt bei Außentemperaturen ab $6^{\circ} \mathrm{C}$ über Rückkühlwerke auf den Dächern der Gebäude. Derzeit trägt der Kältespeicher ca. 50 \% zur Gebäudeklimatisierung im Sommer bei. Daher kommen zusätzlich Kompressions- und Absorptionskältemaschinen, insbesondere für die Niedertemperaturkühlung $\left(6 / 12^{\circ} \mathrm{C}\right)$ zum Einsatz (Kabus und Seibt 2000).

Der Aquifer für die Wärmespeicherung befindet sich in einer Tiefe von ca. $320 \mathrm{~m}$ und ist über eine Brunnendublette erschlossen. Bei überschüssiger Abwärme der betriebenen Blockheizkraftwerke wird der Speicher mit Temperaturen von ca. $70^{\circ} \mathrm{C}$ beladen (Seibt und Kabus 2006). Wiedergewinnungsraten von knapp $80 \%$ ermöglichen Fördertemperaturen von bis zu $65^{\circ} \mathrm{C}$ zu Beginn der Heizperiode (Sanner et al. 2005; Sanner und Knoblich 2004). Allerdings wird die entstehende Abwärme in der Praxis überwiegend für die Absorptionskältemaschinen im Sommer verwendet, sodass der Speicher nur eingeschränkt beladen werden kann. Hinzu kommen aktuell Leckageprobleme in der horizontalen Anbindung, sowie starker Verschleiß der Brunnenpumpen. Dadurch ist der Speicherbetrieb für die Gebäudebeheizung vorläufig stillgelegt. Dennoch konnte ein erfolgreicher Speicherbetrieb sowohl für die Wärme- als auch für

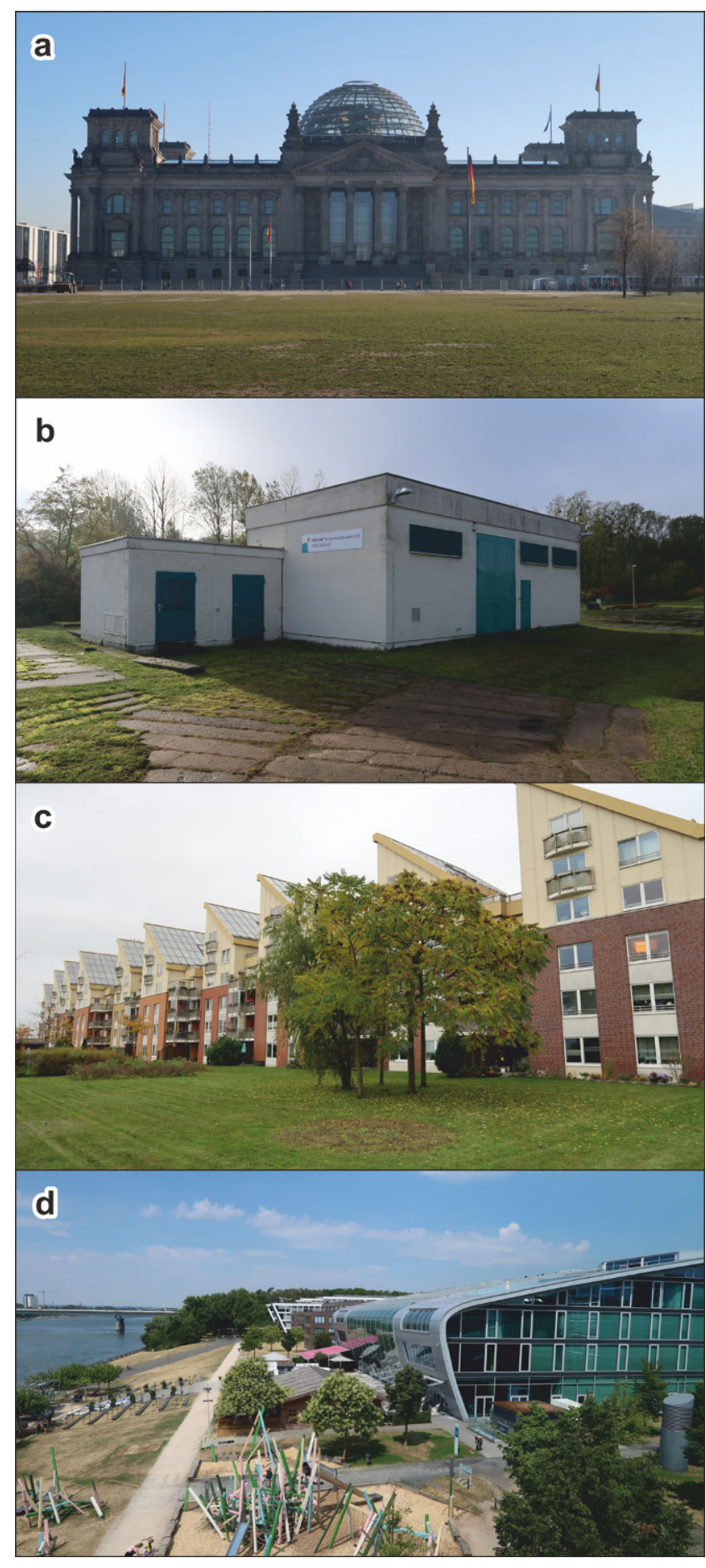

Abb. 3 Fotos realisierter Aquiferspeicherprojekte in Deutschland. a ATES Regierungsgebäude Berlin (@ Schüppler); b HT-ATES Neubrandenburg (@ Fleuchaus); c HT-ATES Rostock-Brinckmanshöhe (@) Fleuchaus); d NT-ATES „Bonner Bogen“ (@ Fleuchaus)

Fig. 3 Pictures of realized German ATES systems. a ATES government buildings Berlin (C Schüppler); b HT-ATES Neubrandenburg (๑) Fleuchaus); c HT-ATES Rostock-Brinckmanshöhe (@ Fleuchaus); d NT-ATES "Bonner Bogen" (@ Fleuchaus) 
Abb. 4 Links:Korrodierte Brunnenpumpe des Aquiferspeichers Neubrandenburg. Rechts: Neuer Kurzzeitspeicher der Neubrandenburger Stadtwerke GmbH (Fleuchaus et al. 2020a)

Fig. 4 Left: Corroded well pump of the cold well of the HT-ATES in Neubrandenburg. Right: New artificial storage tank to balance the short-term supply-demand mismatch (Fleuchaus et al. 2020a)

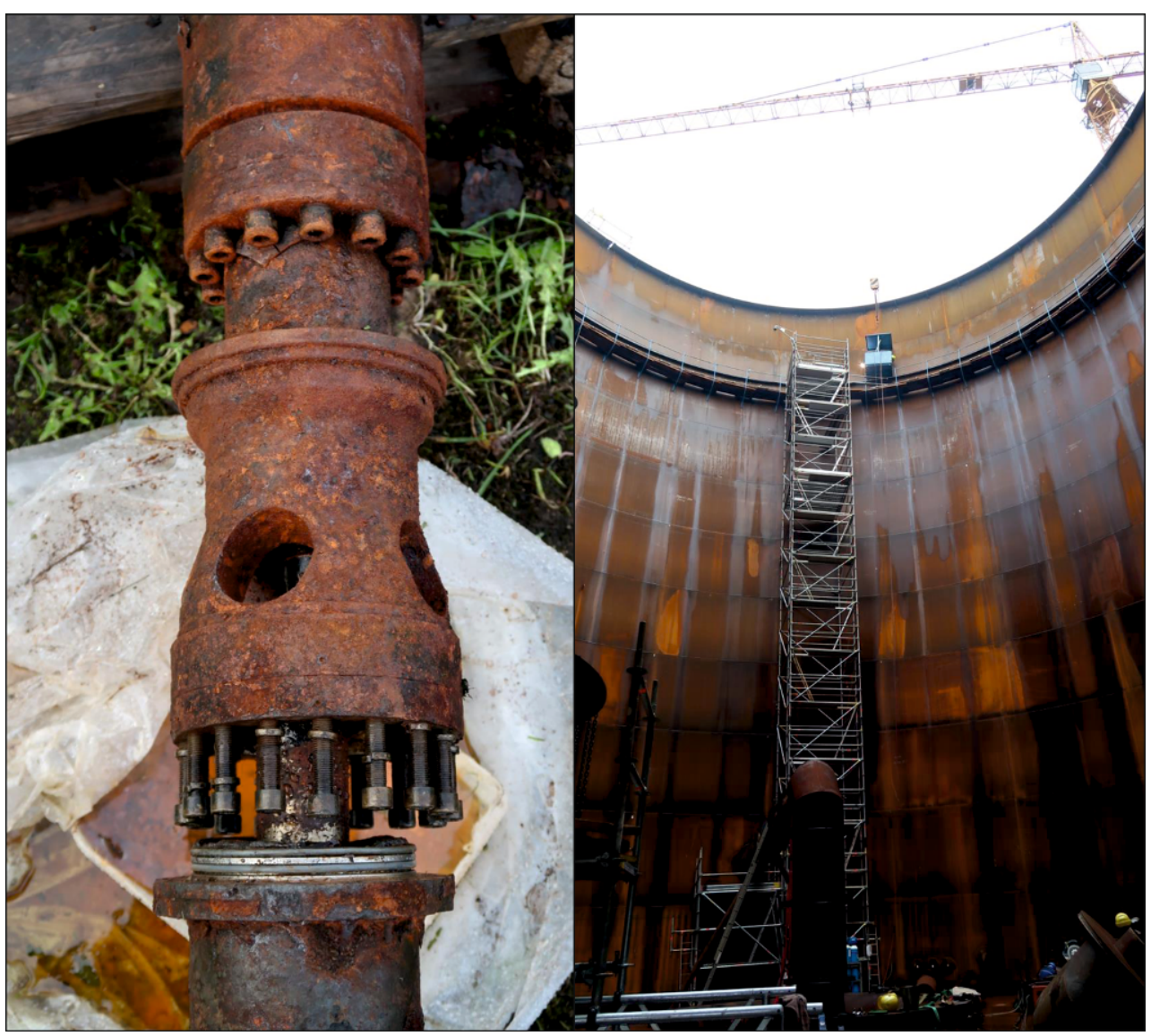

die Kälteversorgung nachhaltig demonstriert werden. Als erster über mehrere Jahre betriebener ATES in Deutschland überhaupt machte das System in Berlin auf die Technologie aufmerksam und dient auch weiterhin als prominentes Anschauungsbeispiel für potenzielle Nachahmer.

\section{Neubrandenburg}

In Neubrandenburg wurde 2005 eine stillgelegte Geothermieanlage reaktiviert, um überschüssige Wärme eines Gasund-Dampf-Kombikraftwerks (GuD) in $1200 \mathrm{~m}$ Tiefe bei einem natürlichen Temperaturniveau von ca. $50^{\circ} \mathrm{C}$ saisonal zu speichern (Abb. 3b). Die gespeicherte Wärme wurde zur Versorgung eines Stadtteils genutzt. Der HT-Speicher war mehr als zehn Jahre in Betrieb, technische Betriebsdaten wurden von Kabus et al. (2004, 2005, 2006 und 2008) publiziert. Technische Probleme traten überwiegend am kalten Brunnen auf, weil Eingabetemperaturen von ca. $30^{\circ} \mathrm{C}$ das Wachstum sulfatreduzierender Bakterien begünstigten (Kabus 2019). Die geochemischen Reaktionen wurden überwacht, analysiert und in mehreren Studien veröffentlicht (Kabus et al. 2009; Würdemann et al. 2014, 2016; Lerm et al. 2013). Obwohl korrodierte Brunnenpumpen regelmäBig ersetzt werden mussten (Abb. 4), wurde der Speicherbetrieb nicht signifikant beeinträchtigt (Kabus 2019).
Trotz hoher (theoretischer) Wiedergewinnungsraten wurde der HT-ATES Anfang 2019 stillgelegt. Starke Schwankungen des Wärmebedarfs und Wärmeangebotes erlaubten keine effiziente Nutzung des Untergrundspeichers ${ }^{1}$. Folglich entschieden sich die Stadtwerke Neubrandenburg für einen Strategiewechsel weg von der saisonalen hin zur Kurzzeitspeicherung. In den Sommermonaten wird in $\mathrm{Zu}-$ kunft die überschüssige Wärme des GuD-Kraftwerkes von Montag bis Freitag in einem künstlichen Stahltank gespeichert. Mit einer Höhe von $36 \mathrm{~m}$ und einem Speichervolumen von $22.000 \mathrm{~m}^{3}$ (Abb. 4) wird die Warmwasserversorgung der Stadt an den Wochenenden gedeckt ${ }^{2}$. Dennoch ist geplant, die vorhandenen Bohrungen für eine (direkte) Geothermieanlage zu reaktivieren (Kabus 2019).

\section{Rostock}

Der einzige HT-Speicher weltweit wird derzeit in RostockBrinckmanshöhe betrieben (Abb. 3c). Mit einer Einspeichertemperatur von $50^{\circ} \mathrm{C}$ befindet sich dieses System aller-

\footnotetext{
${ }^{1}$ Beuster, H.: Persönliche Mitteilung. Neubrandenburger Stadtwerke $\mathrm{GmbH}$ (2019)

2 Beuster, H.: Persönliche Mitteilung. Neubrandenburger Stadtwerke GmbH (2019)
} 
dings im Übergangsbereich zwischen NT- und HT-ATES. Aufgrund des Demonstrationscharakters und hoher Salzkonzentrationen wurde von den lokalen Behörden eine Sondergenehmigung für die Nutzung des oberen Aquifers ( $20 \mathrm{~m}$ Speichertiefe) erteilt (Kabus 2019). Über Sonnenkollektoren eingefangene Wärme wird mithilfe einer Brunnendublette für die winterliche Beheizung des Gebäudekomplexes eingespeichert. Die Beheizung erfolgt in Kombination mit einer Wärmepumpe. Detaillierte Informationen sind in Schmidt und Müller-Steinhagen (2004), Benner et al. (1999) und Bauer et al. (2010) zu finden. Aufgrund der niedrigen Speichertemperaturen traten in über 20 Jahren nur geringfügige technische Probleme auf, wodurch ein reibungsloser Betrieb sichergestellt werden konnte. Aquiferspeicher mit Speichertemperaturen zwischen 35 und $50^{\circ} \mathrm{C}$ haben sich darüber hinaus auch bei vergleichbaren Projekten in den Niederlanden als robuste und aussichtsreiche technische Variante gezeigt (Kallesøe et al. 2019; Drijver et al. 2019).

\section{Bonn}

Seit 2009 versorgt ein NT-ATES das Gebäudeareal „Bonner Bogen" bestehend aus Hotel, Rechenzentrum und Bürokomplexen (Abb. 3d). Sechs Brunnen mit einer Tiefe von maximal knapp $30 \mathrm{~m}$ werden derzeit für die saisonale Wärme- und Kälteversorgung einer Nutzfläche von ca. $60.000 \mathrm{~m}^{2}$ benötigt (Mands et al. 2010). Mit einer genehmigten Fördermenge von bis zu $1.455 .000 \mathrm{~m}^{3} / \mathrm{a}$ zählt das System gleichzeitig zu den größten Wärmepumpenanlagen Europas. Die Beheizung in den Wintermonaten erfolgt in Kombination mit Wärmepumpen mit einem Deckungsanteil von maximal $70 \%$. Für die Deckung der Spitzenlasten stehen Gaskessel zur Verfügung. Das Areal wird im Sommer über die freie Kühlung und Kältemaschinen klimatisiert. Derzeit besteht ein gesteigerter Kältebedarf der Gebäude, u.a. aufgrund der ganzjährigen Kühlung des Rechenzentrums. Daraus ergibt sich ein erhöhter Wärmeeintrag in den Aquifer und ein unausgeglichener Betrieb des Systems von bis zu 70\%, mit negativen Auswirkungen auf Speichereffekte und Effizienz des Gesamtsystems. Aktuell arbeiten die Betreiber des Systems daran den ausgeglichenen Betrieb wiederherzustellen und die Gebäudeanbindung zu optimieren.

\section{Lüneburg}

Der Kreis Bockelsberg in Lüneburg wird mit Wärme aus Biomethan-Blockheizkraftwerken versorgt. Der geplante HT-ATES dient der Minimierung der Wärme aus erdgasbefeuerten Spitzenlastbehältern, um etwa 95\% KWK-Wärme $\mathrm{zu}$ erreichen. Abwärme mit einer Temperatur von bis zu $90^{\circ} \mathrm{C}$ soll in einer Tiefe von ca. $400 \mathrm{~m}\left(20-25^{\circ} \mathrm{C}\right)$ gespei- chert werden (Holstenkamp et al. 2017). Der HT-ATES ist Teil eines Klimaneutralitätskonzeptes der Leuphana Universität (Opel et al. 2017). Trotz intensiver Forschung und Voruntersuchungen, die die technische und wirtschaftliche Machbarkeit des geplanten Systems unterstreichen, ist die Unterstützung für die tatsächliche Umsetzung aufgrund unklarer Risikowahrnehmung lokaler politischer und wirtschaftlicher Entscheidungsträger derzeit gering. Dennoch wird der geplante HT-ATES nach wie vor als eine vielversprechende Option für die zukünftige Entwicklung der Wärmeversorgung Lüneburgs gesehen.

\section{Hamburg}

Im Jahr 2013 haben die Hamburger Bürger im Zuge einer Volksabstimmung die Rekommunalisierung der Energieversorgung der Stadt beschlossen. Der Wiedererwerb des Fernwärmenetzes vom Energiekonzern „Vattenfall Wärme $\mathrm{GmbH}^{\text {“ wurde }} 2019$ abgeschlossen (BUE 2019). Gleichzeitig beschloss die Stadt Hamburg bis 2030 zwei Kohlekraftwerke $(67 \%(1,2 \mathrm{GW}$ thermische Leistung) der momentan gelieferten Wärme) durch weniger $\mathrm{CO}_{2}$-intensive Wärmequellen wie industrielle Abwärme, Kraft-WärmeKopplung oder Abwasser-Wärmerückgewinnung zu ersetzen. Die Flexibilität des neuen Fernwärmenetzes soll zudem durch die Integration von Kurz- als auch Langzeitwärmespeichern erhöht werden. HT-ATES wird als Schlüsseltechnologie betrachtet und potenzielle Wärmequellen, unterschiedliche Speicherhorizonte sowie eine Integration in das Wärmenetz werden derzeit untersucht. Als aussichtsreiche Speicherhorizonte gelten die „Oberen Braunkohlesande" (OBKS) in einer Tiefe von 200-300 m und eine $1000 \mathrm{~m}$ tiefe Sandsteinformation ${ }^{3}$ (Radmann und Hansen 2019). Im Jahr 2017 wurde auf der Elbinsel Dradenau ein Testbrunnen für einen zyklischen Speicherbetrieb installiert. Mit einem Rückgewinnungsgrad von rund $90 \%$ konnte die technische Machbarkeit der Wärmespeicherung im UBKS erfolgreich demonstriert werden (Radmann und Hansen 2019). Zudem werden derzeit unterschiedliche Speicherorte sowie verschiedene Wärmequellen und -senken untersucht. In diesem Zusammenhang wurde das Projekt IW3 aus dem Programm „Living lab“ des Bundesministeriums für Wirtschaft und Energie (BMWI) gefördert. Das Projekt baut auf den Voruntersuchungen der Firma „GTW Geothermie Wilhelmsburg GmbH“" auf, die die Tiefengeothermie (3-4km) im Raum Hamburg untersucht. Ziel von IW3 ist der Aufbau einer dezentralen, $\mathrm{CO}_{2}$-neutralen Wärmeversorgung für den Stadtteil Wilhelmsburg. Für eine optimale Integration unterschiedlicher Wärmequellen (Tiefengeothermie, industrielle Abwärme, Power-to-Heat) ist die Errichtung eines HT-ATES geplant.

\footnotetext{
${ }^{3}$ Radmann, K.J.: Persönliche Mitteilung (2019)
} 


\section{SWOT-Analyse}

Im Zuge einer SWOT-Analyse haben wir im folgenden Kapitel alle relevanten internen und externen Faktoren identifiziert, die die ATES-Entwicklung auf dem deutschen Energiemarkt beeinflussen. Die einzelnen Faktoren werden in Stärken (S), Schwächen (W), Chancen (O) und Risiken (T) gruppiert. Chancen sind Möglichkeiten, neue Märkte im Bereich der Energieversorgung zu erschließen. Diese Chancen können durch alternative Versorgungstechnologien oder durch technologische und wirtschaftspolitische Veränderungen gefährdet werden (Risiken). Zur Vermeidung der Risiken werden Strategien durch Einschätzung der eigenen Stärken und Schwächen (im Vergleich zu alternativen Technologien) entwickelt. Abb. 5 skizziert die Ergebnisse der SWOT-Analyse unterteilt nach HT- und NT-ATES. Die wichtigsten Punkte werden im Folgenden diskutiert.

\section{NT-ATES}

NT-ATES wird derzeit überwiegend in den Niederlanden betrieben. Dies ist zum einen auf eine aktive Förderung durch die niederländische Regierung, aber auch durch flächendeckend geringe Grundwasserfließgeschwindigkeiten von lokal weniger als $1 \mathrm{~m} / \mathrm{a}$ zurückzuführen, die hohe Wiedergewinnungsraten begünstigt. Obwohl sich aus der aktiven Wärme- und Kältespeicherung Effizienzvorteile aufgrund optimierter Fördertemperaturen ergeben (Abb. 5), werden in Deutschland fast ausnahmslos klas- sische Brunnenanlagen mit Grundwasserwärmepumpen (GWWP) betrieben. Im Vergleich zu GWWP erlauben NT-ATES Systeme aufgrund des Speichereffekts erhöhte Entnahmetemperaturen zwischen 3 und $10 \mathrm{~K}$ gegenüber der natürlichen Grundwassertemperatur im Winter, in Abhängigkeit der Einspeisetemperaturen im Sommer und der auftretenden Wärmeverluste während der Speicherperiode. Als besonders effektiv erweisen sich NT-ATES bei der Gebäudeklimatisierung im Sommer, da die geringeren Grundwassertemperaturen eine freie Kühlung ohne Zuschaltung der Wärmepumpe ermöglichen. Dies ist insbesondere in großen Stadtzentren von Vorteil, wo der Effekt der urbanen Wärmeinsel mit stark erhöhten Grundwassertemperaturen eine direkte Grundwassernutzung zur Kälteversorgung erschwert. Ein sehr hohes Potenzial für die NT-ATES Nutzung bietet sich insbesondere im Norden Deutschlands, im Bereich des Oberrheingrabens sowie im Molassebecken des Alpenvorlandes. Hohe finanzielle Einsparungen insbesondere im Vergleich zu herkömmlichen Technologien konnten bereits durch Schüppler et al. (2019) in einer Fallstudie für das Städtische Klinikum Karlsruhe (SKK) demonstriert werden. Für finanzielle und energetische Einsparungen bedarf es aber nicht nur einer optimalen Planung des ATES selbst, sondern auch einer fachgerechten Integration der Untergrundkomponenten in das Heiz- und Kühlsystem (HVAC) des Gebäudes. Studien aus den Niederlanden haben jedoch gezeigt, dass lediglich $30 \%$ der installierten Systeme einen optimalen Betrieb aufweisen. Während hohe Wiedergewinnungsraten beobachtet

\begin{tabular}{|c|c|c|c|c|}
\hline & 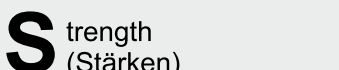 & $\begin{array}{l}\text { eaknesses } \\
\text { (Schwächen) }\end{array}$ & & $\begin{array}{l}\text { hreats } \\
\text { (Risiken) }\end{array}$ \\
\hline $\begin{array}{l}\text { NT } \\
\text { S } \\
\bar{E} \\
\bar{E}\end{array}$ & $\begin{array}{l}\text { - Kurze Amortisierung } \\
\text { - Hohe } \mathrm{CO}_{2} \text {-Einsparungen } \\
\text { - Heizen und Kühlen } \\
\text { - Freie Kühlung möglich } \\
\text { - Geringer Platzbedarf }\end{array}$ & $\begin{array}{l}\text { - Nur im NT-Sektor } \\
\text { anwendbar } \\
\text { - Hohe Investitionskosten } \\
\text { - Wartungsintensiv } \\
\text { - Nur NT-Wärmequellen } \\
\text { - Temperaturgrenzwerte } \\
\text { - Langwieriges/aufwändiges } \\
\text { Genehmigungsverfahren }\end{array}$ & $\begin{array}{l}\text { - Sehr hohe Speicherkapazität } \\
\text { - Sehr hoher Wärme- } \\
\text { überschuss } \\
\text { - Kombination mit erneuer- } \\
\text { baren Energien }\end{array}$ & $\begin{array}{l}\text { - Abhängigkeit von Rand- } \\
\text { bedingungen }\left(\mathrm{CO}_{2} \text {-Preis, }\right. \\
\text { Strompreis, Wärme- und } \\
\text { Kältenachfrage) } \\
\text { - TGA-Planung }\end{array}$ \\
\hline $\begin{array}{l}\text { HT } \\
\qquad \overline{\bar{E}} \\
\\
\end{array}$ & $\begin{array}{l}\text { - Hohe Energiedichte } \\
\text { - Versorgung von HT- } \\
\text { Anwendungen } \\
\text { - Geringer Platzbedarf }\end{array}$ & $\begin{array}{l}\text { - Geringe Flexibilität } \\
\text { - Abhängig von Wärmequelle } \\
\text { und -senke } \\
\text { - Hohe Investitionskosten } \\
\text { - Wartungsintensiv } \\
\text { - Umfangreiches Monitoring } \\
\text { - Langwieriges/aufwändiges } \\
\text { Genehmigungsverfahren }\end{array}$ & $\begin{array}{l}\text { - Sehr hohe Speicherkapazität } \\
\text { - Unabhängigkeit von } \\
\text { externen Wärmequellen } \\
\text { - Kombination mit } \\
\text { erneuerbaren Energien } \\
\text { - Steigender Kältebedarf }\end{array}$ & $\begin{array}{l}\text { - Technische Risiken } \\
\text { (Geochemie, TGA-Planung) } \\
\text { - Widersprüchliche Interessen } \\
\text { (Klima- und Grundwasser- } \\
\text { schutz) } \\
\text { - Abhängigkeit von Rand- } \\
\text { bedingungen }\left(\mathrm{CO}_{2} \text {-Preis, }\right. \\
\text { Strompreis, Wärme- } \\
\text { nachfrage, Wärmeangebot) }\end{array}$ \\
\hline
\end{tabular}

Abb. 5 Übersicht der SWOT-Analyse für Aquiferspeicher in Deutschland

Fig. 5 Overview of the SWOT-analysis of ATES application in Germany 
werden, sind geringe Jahresarbeitszahlen der Systeme auf ineffiziente Gebäudekomponenten, ein fehlendes Gebäudemonitoring oder ungenaue/fehlende Bedarfsermittlungen zurückzuführen (Fleuchaus et al. 2020b). Ähnliche Probleme können auch in Deutschland beobachtet werden, wo fehlende Fachkenntnisse im Bereich der TGA (Technische Gebäudeausrüstung)-Planung eine kritische Marktbarriere für eine Kommerzialisierung der Technologie darstellen (Fleuchaus et al. 2018).

\section{HT-ATES}

HT-ATES zeichnen sich im Vergleich zu NT-Systemen durch höhere Speicher- und Fördertemperaturen und damit verbunden höhere Speicherkapazitäten aus. Die HTSpeicherung ist nicht nur auf den NT-Gebäudesektor beschränkt, sondern erlaubt auch die Versorgung von Fernwärmenetzen oder Wärmeabnehmern aus der Industrie oder von Gewächshäusern. Im Vergleich zur direkten tiefengeothermischen Nutzung werden zudem deutlich flachere Förderhorizonte benötigt, wodurch sich die Bohrkosten erheblich reduzieren. HT-ATES bieten somit ein großes (ungenutztes) Potenzial, erneuerbare Energien sowie derzeit meist ungenutzte industrielle Abwärme flächendeckend als Wärmequellen in den thermischen Energiesektor zu integrieren. Besonders im Vergleich zu NT-ATES sind die höheren Speichertemperaturen allerdings auch mit technischen, legislativen und politischen Risiken verbunden (Fleuchaus 2020). Diese sind stark abhängig von den vorherrschenden Randbedingungen, welche sich zum Teil auf regionaler und lokaler Ebene unterscheiden. Dies gilt nicht nur für die Eigenschaften des Untergrundes, sondern beispielsweise auch für die Stabilität der Wärme- und Kältequelle oder der Offenheit des zuständigen Landratsamtes für innovative, in Deutschland nicht erprobte Technologien. Während im Südwesten Deutschlands noch immer große Vorbehalte in der Bevölkerung gegen die geothermische Nutzung des Untergrundes vorherrschen, konnte in Hamburg oder Neubrandenburg eine hohe Zustimmung für Pilotversuche im Bereich der Aquiferspeicherung beobachtet werden ${ }^{4}$ (Kabus 2019). Risiken und Barrieren der Aquiferspeicherung weltweit sind ausführlich in Fleuchaus (2020), Fleuchaus et al. (2018) und (2020b) beschrieben.

Mit Speichertemperaturen zwischen 70 und $120^{\circ} \mathrm{C}$ haben geochemische Prozesse im Untergrund in der Vergangenheit häufig zu Problemen wie Verockerung oder Korrosion geführt, konnten aber durch langjährige Forschung (Lerm et al. 2011; Bonte et al. 2013; Jesußek et al. 2013; Lienen et al. 2017; Ueckert und Baumann 2019) und entwickelte Gegenmaßnahmen überwiegend kontrolliert werden. Vergangene und aktuelle HT-ATES-Projekte haben dage-

\footnotetext{
${ }^{4}$ Radmann, K.J.: Persönliche Mitteilung (2019)
}

gen gezeigt, dass die größte Herausforderung in der Trägheit der Systeme in Verbindung mit saisonalen Schwankungen des Energieangebots und der Energienachfrage sowie in den sich ändernden Nutzungskonzepten liegt. Zudem muss über die gesamte Nutzungsdauer von bis zu 50 Jahren eine stetig verfügbare Wärmequelle gesichert sein. HT-ATESProjekte insbesondere in den Niederlanden und Deutschland haben gezeigt, dass dies in der Praxis aufgrund sich ändernder Randbedingungen (z.B. politische Fördermaßnahmen, Energiekosten, Nutzungsverhalten) oftmals nicht gegeben ist. Dies konnte kürzlich in Neubrandenburg beobachtet werden (Abb. 3b), wo ein saisonaler HT-ATES durch einen künstlichen Kurzzeitspeicher ersetzt wurde (Abb. 4). Für eine flächendeckende HT-ATES-Nutzung in Deutschland ist deshalb nicht nur eine umfassende Planung, Auslegung und Überwachung der Systeme, sondern auch die Schaffung optimaler Randbedingungen entscheidend. Dies umfasst nicht nur eine Vereinfachung genehmigungsrechtlicher Vorgaben und Rahmenbedingungen, sondern auch eine aktive Förderung der Aquiferspeicherung in Deutschland durch die Schaffung neuer und langfristiger Anreizprogramme sowie die frühzeitige Einbindung der Öffentlichkeit. In den Niederlanden lässt sich über ein vom Ministerium für Wirtschaft und Klima bereitgestelltes GIS-basiertes Webtool für jeden einfach nachvollziehen, ob sich ein Aquiferspeicher am vorgesehenen Standort realisieren lässt oder ob bestimmte Einschränkungen, wie z.B. Grundwasserschutzgebiete, einer Umsetzung im Wege stehen (Ministerie van Economische Zaken en Klimaat). Dadurch lässt sich frühzeitig eine theoretische technische Machbarkeit abschätzen und der Bekanntheitsgrad der Technologie deutlich erhöhen. Darüber hinaus ist die Gesetzgebung in den Niederlanden sowohl auf Untergrundspeicher als auch konkret auf Aquiferspeicher (Wasserrecht) angepasst, wodurch die Genehmigungsverfahren einfacher nachvollziehbar und verkürzt sind (ca. 6 Monate) (Bloemendal et al. 2016; Kallesøe und Vangkilde-Pedersen 2019). Ein einheitliches Genehmigungsverfahren unabhängig von den Ländergrenzen wäre auch in Deutschland für eine vermehrte Umsetzung der Technologie förderlich. Zudem sollten zukünftig positive Auswirkungen eines Vorhabens auf den Klimaschutz seitens der Behörden berücksichtigt werden (Degenhart et al. 2019). Dies betrifft nicht nur die $\mathrm{CO}_{2}$-Vermeidung, sondern auch die Nutzung des anthropogenen Wärmeeintrags in urbanen Räumen.

Begünstigte Randbedingungen für saisonale Speicher sind auch in Dänemark anzutreffen. Eine höhere Besteuerung fossiler Brennstoffe, weite Verbreitung von Wärmenetzen sowie langfristig angelegte und verbindliche kommunale Wärmeplanung zählen zu den Hauptfaktoren erfolgreicher Projekte und würden die Aquiferspeicherung auch in Deutschland vorantreiben (Pehnt 2017). Aktuell sind die Fördermöglichkeiten für Speichertechnologien in 
Deutschland, die über Machbarkeitsstudien hinausgehen, noch gering. Mit der Bundesförderung für effiziente Wärmenetze (Wärmenetze 4.0) wird neuerdings jedoch auch die Realisierung eines innovativen Wärmenetzsystems in Kombination mit saisonaler Wärmespeicherung gefördert (Bundesamt für Wirtschaft und Ausfuhrkontrolle (BAFA) 2019). Zudem besteht die Möglichkeit über wissenschaftliche Begleitung und intensive Monitoring-Fördermöglichkeiten zu generieren. Zukünftige Einnahmen aus der $\mathrm{CO}_{2}-$ Bepreisung könnten nach Renn et al. (2019) ganz gezielt für neue Forschungs- und Markteinführungsprogramme von Speichern verwendet werden. Zusammenfassend lässt sich festhalten, dass HT-ATES im Vergleich zu konkurrierenden Technologien (Beckenspeicher, Tiefengeothermie, NTATES) einen erheblichen Beitrag zur Speicherung erneuerbarer HT-Wärmequellen leisten kann und in Abhängigkeit der lokalen Randbedingungen ein enormes Marktpotenzial aufweist. Erfahrungen aus der Tiefengeothermie zeigen, dass die technische Robustheit und Kosteneffizienz auch im Dauerbetrieb demonstriert werden muss, um das Vertrauen von Investoren und Politik zu gewinnen. Die Umsetzung tiefengeothermischer Systeme in der Stadt München zeigt, dass den vielfältigen Risiken der HT-ATES durch ein umfassendes Energiekonzept und eine frühzeitige Einbindung der Öffentlichkeit begegnet werden kann. Erfahrungen aus der Windkraftbranche haben zudem bewiesen, dass die öffentliche Akzeptanz eines Projekts nicht wie oft nur auf einer frühzeitigen und umfassenden Informationspolitik basieren müssen. Eine finanzielle Beteiligung umliegender Gemeinden oder Stadtteile durch die Bildung neuer Bürgerenergiegenossenschaften und damit Rekommunalisierung der Wärmeversorgung ließe sich in Kombination moderner Wärme- und Kältenetze effektiv auf die Aquiferspeichertechnologie übertragen. Hierbei müssen allerdings die sich kurz- und langfristig ändernden Randbedingungen sowie die Wirtschaftlichkeit der Systeme berücksichtigt werden (Fleuchaus et al. 2020a).

\section{Fazit}

Mit über 50 Jahren Forschungsaktivität im Bereich der Aquiferspeicherung konnte in Deutschland ein wichtiger Beitrag zur Weiterentwicklung der Technologie geleistet werden. Trotz des langjährigen Betriebes innovativer ATES-Anlagen in Bonn, Neubrandenburg, Rostock und insbesondere Berlin konnte sich die Technologie bisher allerdings nicht auf dem Energiemarkt durchsetzen, was nicht zuletzt auf die meist unzureichende Darlegung und Dokumentation der technischen Betriebsdaten und wirtschaftlichen Potenziale der Systeme zurückzuführen ist. Ambitionierte Klimaschutzmaßnahmen, die Schwierigkeit der Integration erneuerbarer Energien in die Wärme- und
Kälteversorgung sowie ein großes geologisches Potenzial in Deutschland fordern innovative und zukunftsträchtige Lösungen bei der energetischen Versorgung von Gebäuden und eröffnen somit einen neuen Wachstumsmarkt für ATES in Deutschland. Für eine erfolgreiche Kommerzialisierung am Markt gilt es in Zukunft die folgenden Punkte zu beachten:

- Die Errichtung weiterer Demonstrationsanlagen dient nicht nur der technischen Weiterentwicklung, sondern erhöht auch den Bekanntheitsgrad und die Akzeptanz der Technologie unter Entscheidungsträgern aus Politik, Gebäudeplanung und Energiewirtschaft. Zukünftige Demonstrationsprojekte sollten daher nicht nur technische, hydraulische und hydrochemische Daten dokumentieren, sondern insbesondere auch finanzielle Aspekte und Risikoanalysen verstärkt berücksichtigen (Fleuchaus et al. 2018).

- Für eine schnelle und kosteneffektive Realisierung weiterer HT- und NT-Systeme bedarf es einer Vereinfachung der genehmigungsrechtlichen Anforderungen (Fleuchaus 2020); Der Einfluss von Speichertemperaturen über $20^{\circ} \mathrm{C}$ auf die Ökologie und Geochemie des Grundwassers muss zudem weiter erforscht werden. Dadurch lassen sich gegebenenfalls lokal die Anforderungen anpassen, insbesondere unter der Berücksichtigung, dass einzelne Infrastrukturen im urbanisierten Raum das Grundwasser bereits deutlicher erwärmen (Tissen et al. 2019).

- Bereits jetzt realisierte Anreizprogramme und Fördermaßnahmen stellen eine vielversprechende Grundlage für die Umsetzung von NT- und HT-ATES in Deutschland dar, beschränken sich allerdings oftmals nur auf die Durchführung von Machbarkeitsstudien. Daher gilt es seitens der Politik entsprechende Programme auszuweiten, diese spezifisch auf Speichertechnologien anzupassen sowie diese schlussendlich transparent zu kommunizieren und den Zugang zu erleichtern.

- Risikoanalysen werden in vielen Bereichen der Energieversorgung bereits angewendet und sind für Projektverantwortliche entscheidend, um auftretende Risiken abzuschwächen und Projektziele zu erreichen. Im Bereich der geothermischen Nutzung findet die Untersuchung von Risiken jedoch oftmals wenig Beachtung, obwohl insbesondere bei der Nutzung HT-ATES der Bedarf dafür groß ist. Daher müssen zukünftig geeignete Methoden entwickelt und Projektbeteiligte für deren Bedeutung sensibilisiert werden. Neben den technischen Risiken muss zudem ein besonderes Augenmerk auch auf legislative, soziale und politische Risiken gelegt werden.

Danksagung Wir bedanken uns beim Ministerium für Umwelt, Klima und Energiewirtschaft Baden-Württemberg für die Förderung des Forschungsprojekts Geospeicher.bw im Rahmen von BWPLUS (För- 
derkennzeichen L7516014-16019) sowie allen beteiligten Projektpartnern. Für wertvolle Anregungen und kritische Kommentare danken wir zwei anonymen Reviewern.

Funding Open Access funding enabled and organized by Projekt DEAL.

Open Access Dieser Artikel wird unter der Creative Commons Namensnennung 4.0 International Lizenz veröffentlicht, welche die Nutzung, Vervielfältigung, Bearbeitung, Verbreitung und Wiedergabe in jeglichem Medium und Format erlaubt, sofern Sie den/die ursprünglichen Autor(en) und die Quelle ordnungsgemäß nennen, einen Link zur Creative Commons Lizenz beifügen und angeben, ob Änderungen vorgenommen wurden.

Die in diesem Artikel enthaltenen Bilder und sonstiges Drittmaterial unterliegen ebenfalls der genannten Creative Commons Lizenz, sofern sich aus der Abbildungslegende nichts anderes ergibt. Sofern das betreffende Material nicht unter der genannten Creative Commons Lizenz steht und die betreffende Handlung nicht nach gesetzlichen Vorschriften erlaubt ist, ist für die oben aufgeführten Weiterverwendungen des Materials die Einwilligung des jeweiligen Rechteinhabers einzuholen.

Weitere Details zur Lizenz entnehmen Sie bitte der Lizenzinformation auf http://creativecommons.org/licenses/by/4.0/deed.de.

\section{Literatur}

Adinolfi, M., Koch, M., Ruck, W.K.L.: Ökologische und mikrobielle Folgen der Wärmespeicherung im Aquifer. In: Saisonale Wärmespeicher im Aquifer: Chancen und Risiken für die Umwelt Stuttgarter Berichte zur Siedlungswasserwirtschaft, Bd. 124, Oldenbourg, München (1994)

Adinolfl, M., Ruck, W.K.L.: Microbiological and environmental effects of aquifer thermal energy storage-Studies at the Stuttgart man-made aquifer and a large-scale model system. In: Jenne, E.A. (Hrsg.) Aquifer Thermal Energy (Heat and Chill) Storage: Proceedings of the 27th Intersociety Energy Conversion Engineering Conference, San Diego, CA, S. 69-81. (1992)

Andersson, O.: Aquifer Thermal Energy Storage (ATES). In: Paksoy, H.Ö. (Hrsg.) Thermal Energy Storage for Sustainable Energy Consumption, S. 155-176. Springer Netherlands, Dordrecht (2007)

Bakema, G., Snijders, A., Nordell, B.: Underground Thermal Energy Storage: State of the Art 1994. International Energy Agency (IEA), Paris (1995)

Bauer, D., Marx, R., Nußbicker-Lux, J., Ochs, F., Heidemann, W., Müller-Steinhagen, H.: German central solar heating plants with seasonal heat storage. Sol Energy 84(4), 612-623 (2010). https://doi. org/10.1016/j.solener.2009.05.013

Benner, M., Mahler, B., Mangold, D., Schmidt, T., Schulz, M., Seiwald, H.: Solar unterstützte Nahwärmeversorgung mit und ohne Langzeit-Wärmespeicher: Forschungsbericht zum BMBF-Vorhaben (September 1994 bis Oktober 1998). Universitätsbibliothek, Technische Informationsbibliothek, Hannover, Stuttgart, S. 103 (1999)

Bloemendal, M., Hoekstra, N., Slenders, H., van de Mark, B., van de Ven, F., Andreu, A., Simmons, N., Sani, D.: Europe-Wide Use of Sustainable Energy from Aquifers. Barrier Assessment (2016)

Bloemendal, M., Olsthoorn, T., van de Ven, F.: Combining climatic and geo-hydrological preconditions as a method to determine world potential for aquifer thermal energy storage. Sci. Total Environ. 538, 621-633 (2015). https://doi.org/10.1016/j.scitotenv.2015.07. 084
Bloemendal, M.: The hidden side of cities: Methods for governance, planning and design for optimal use of subsurface space with ATES. Dissertation, TU Delft (2018)

Bonte, M., van Breukelen, B.M., Stuyfzand, P.J.: Temperature-induced impacts on groundwater quality and arsenic mobility in anoxic aquifer sediments used for both drinking water and shallow geothermal energy production. Water Res. 47(14), 5088-5100 (2013). https://doi.org/10.1016/j.watres.2013.05.049

BUE: Wärmewande \& Energiepark Hafen. Behörde für Umwelt und Energie. https://www.hamburg.de/contentblob/12957152/ 7374b07373873dce6dd7af51f012383c/data/d-waermewende.pdf (2019). Zugegriffen: 19. Dez. 2019

Bundesamt für Wirtschaft und Ausfuhrkontrolle (BAFA): Bundesförderung für effiziente Wärmenetze (2019). https://www.bafa.de/ DE/Energie/Energieeffizienz/Waermenetze/waermenetze_node. html, Zugegriffen: 19. Okt. 2020

Degenhart, H., Holstenkamp, L., Kohrs, A.K., Neidig, P., Opel, O., Schomerus, T., Strodel, N., Michalzik, D., Steffahn, J., Porcello, K., Meisel, M., Weber, J., Schönebeck, J., Kruck, O.: Schlussbericht zum Forschungsvorhaben Aquiferspeicher (2019). Entwicklung der Einsatzfelder für mitteltiefe Aquiferwärmespeicher in Norddeutschland unter wirtschaftlich/finanziellen, geologisch/ technischen, umweltchemischen und rechtlich/förderpolitischen Aspekten

Dickinson, J.S., Buik, N., Matthews, M.C., Snijders, A.: Aquifer thermal energy storage: theoretical and operational analysis. Géotechnique 59(3), 249-260 (2009). https://doi.org/10.1680/geot.2009. 59.3.249

Drijver, B., Bakema, G., Oerlemans, P.: State of the art of HT-ATES in The Netherlands. In: European Geothermal Congress: Proceedings, Den Haag, Netherlands (2019)

Fleuchaus, P.: Global application, risk and performance analysis of Aquifer Thermal Energy Storage (ATES). Dissertation, Karlsruhe Institute of Technology (KIT) (2020)

Fleuchaus, P., Godschalk, B., Stober, I., Blum, P.: Worldwide application of aquifer thermal energy storage-A review. Renew Sustain. Energy Rev. 94, 861-876 (2018). https://doi.org/10.1016/j.rser. 2018.06.057

Fleuchaus, P., Schüppler, S., Bloemendal, M., Guglielmetti, L., Opel, O., Blum, P.: Risk analysis of High-Temperature Aquifer Thermal Energy Storage (HT-ATES). Renew. Sustain. Energy Rev. (2020a). https://doi.org/10.1016/j.rser.2020.110153

Fleuchaus, P., Schüppler, S., Godschalk, B., Bakema, G., Blum, P.: Performance analysis of Aquifer Thermal Energy Storage (ATES). Renew. Energy 146, 1536-1548 (2020b). https://doi.org/10.1016/ j.renene.2019.07.030

Holstenkamp, L., Meisel, M., Neidig, P., Opel, O., Steffahn, J., Strodel, N., Lauer, J.J., Vogel, M., Degenhart, H., Michalzik, D., Schomerus, T., Schönebeck, J., Növig, T.: Interdisciplinary review of medium-deep aquifer thermal energy storage in north Germany. Energy Procedia 135, 327-336 (2017). https://doi.org/10.1016/j. egypro.2017.09.524

Jenne, E.A., Andersson, O., Willemsen, A.: Hydrogeologische und geotechnische Aspekte der Wärmespeicherung im Aquifer-potenzielle Standorte. In: Jenne, E.A. (Hrsg.) Aquifer Thermal Energy (Heat and Chill) Storage: Proceedings of the 27th Intersociety Energy Conversion Engineering Conference, San Diego, CA (1992) https://doi.org/10.4271/929153

Jesußek, A., Köber, R., Grandel, S., Dahmke, A.: Aquifer heat storage: sulphate reduction with acetate at increased temperatures. Environ Earth Sci 69(5), 1763-1771 (2013). https://doi.org/10.1007/ s12665-012-2009-0

Kabus, F.: Persönliche Mitteilung. Geothermie Neubrandenburg (GTN) (2019)

Kabus, F., Seibt, P.: Aquifer Thermal Energy Storage for the Berlin Reichstag Building-New Seat of the German Parliament. Proceedings World Geothermal Congress, S. 3611-3615. Inter- 
national Geothermal Association (IGA), Kyushu-Tohoku, Japan (2000)

Kabus, F., Möllmann, G., Hoffmann, F.: Speicherung von Überschusswärme aus dem Gas- und Dampfturbinen-Heizkraftwerk. In: GTV-Tagung, Landau, Germany (2004)

Kabus, F., Hoffmann, F., Möllmann, G.: Aquifer storage of waste heat arising from a gas and steam cogeneration plant-Concept and first operating experience. In: Proceedings World Geothermal Congress, Antalya, Turkey (2005)

Kabus, F., Richlak, U., Beuster, H.: Saisonale Speicherung von Überschusswärme aus einem Heizkraftwerk in einem Aquifer in Neubrandenburg. In: Statusseminar „Thermische Energiespeicherung" Freiburg, Germany (2006)

Kabus, F., Richlak, U., Wolfgramm, M., Gehrke, D., Beuster, H., Seibt, A.: Aquiferspeicher Neubrandenburg - Betriebserfahrung über drei Speicherzyklen. In: Der Geothermiekongress, Karlsruhe, Germany (2008)

Kabus, F., Wolfgramm, M., Seibt, A., Richlak, U., Beuster, H.: Aquifer thermal energy storage in Neubrandenburg-Monitoring throughout three years of regular operation. In: Proceedings Effstock. 11th International Conference on Thermal Energy Storage for Energy Efficiency and Sustainability, Stockholm, Sweden (2009)

Kallesøe, A.J., Vangkilde-Pedersen, T.: Underground Thermal Energy Storage (UTES) - State-of-the-Art, Example Cases and Lessons Learned: HEATSTORE Project Report (2019)

Kranz, S., Frick, S.: Efficient cooling energy supply with aquifer thermal energy storages. Applied Energy 109, 321-327 (2013). https://doi.org/10.1016/j.apenergy.2012.12.002

Kranz, S., Bartels, J., Gehrke, D., Hoffmann, F., Wolfgramm, M.: Wärme- und Kältespeicherung in Aquiferen. bbr 59, 34-43 (2008)

Kunkel, C., Agemar, T., Stober, I.: Geothermisches Nutzungspotenzial der Buntsandstein- und Keuperaquifere im Nordosten Bayerns mit Fokus auf tiefe Aquiferspeicher. Grundwasser 24(4), 251-267 (2019). https://doi.org/10.1007/s00767-019-00430-1

Lerm, S., Alawi, M., Miethling-Graff, R., Wolfgramm, M., Rauppach, K., Seibt, A., Würdemann, H.: Influence of microbial processes on the operation of a cold store in a shallow aquifer: Impact on well injectivity and filter lifetime. Grundwasser 16(2), 93-104 (2011). https://doi.org/10.1007/s00767-011-0165-x

Lerm, S., Westphal, A., Miethling-Graff, R., Alawi, M., Seibt, A., Wolfgramm, M., Würdemann, H.: Thermal effects on microbial composition and microbiologically induced corrosion and mineral precipitation affecting operation of a geothermal plant in a deep saline aquifer. Extremophiles 17(2), 311-327 (2013). https://doi.org/10.1007/s00792-013-0518-8

Lienen, T., Lüders, K., Halm, H., Westphal, A., Köber, R., Würdemann, H.: Effects of thermal energy storage on shallow aerobic aquifer systems: Temporary increase in abundance and activity of sulfate-reducing and sulfur-oxidizing bacteria. Environ Earth Sci 76(6), 403 (2017). https://doi.org/10.1007/s12665-017-6575-z

Lu, H., Tian, P., He, L.: Evaluating the global potential of aquifer thermal energy storage and determining the potential worldwide hotspots driven by socio-economic, geo-hydrologic and climatic conditions. Renew. Sustain. Energy Rev. 112, 788-796 (2019). https://doi.org/10.1016/j.rser.2019.06.013

Mands, E., Sanner, B., Sauer, M., Grundmann, E., Brehm, D.: Grundwassergekoppelte Wärmepumpeanlage am Bonner Bogen. bbr 2010(Sonderheft 2010), 74-80 (2010)

Mangold, D., Miedaner, O., Tziggili, E.P., Schmidt, T., Unterberger, M., Zeh, B.: Technisch-Wirtschaftliche Analyse und Weiterentwicklung der solaren Langzeit-Wärmespeicherung. SOLITES (2012). http://www.solites.de/download/literatur/Solites_Techn isch-wirtschaftliche\%20Analyse\%20und\%20Weiterentwicklung $\% 20$ der\%20solaren\%20Langzeit-Waermespeicherung_Forschung sbericht_FKZ\%200329607N_2012.pdf. Zugegriffen: 10. Mai 2020
Mercer, J.W., Faust, C.R., Miller, W.J., Pearson Jr., F.J.: Review of Simulation Techniques for Aquifer Thermal Energy Storage (ATES). Pacific Northwest Laboratory, Pacific Northwest Lab., Richland, WA (USA) (1981)

Opel, O., Strodel, N., Werner, K.F., Geffken, J., Tribel, A., Ruck, W.: Climate-neutral and sustainable campus Leuphana University of Lueneburg. Energy 141, 2628-2639 (2017). https://doi.org/10. 1016/j.energy.2017.08.039

Pehnt, M.: Wärmenetze 4.0: Endbericht - Kurzstudie zur Umsetzung der Maßnahme „Modellvorhaben erneuerbare Energien in hocheffizienten Niedertemperaturwärmenetzen“ (2017)

Radmann, K.J., Hansen, C.: Erneuerbare Wärme in Hamburg: Installation eines Aquifer-Wärmespeichers. In: Berliner Energietage, Berlin, Germany (2019)

Renn, O., Becker, S., Gaschnig, H., Götting, K., Lilliestam, J., Schäbule, D., Setton, D.: $\mathrm{CO}_{2}$-Bepreisung für eine sozial gerechte Energiewende. IASS Policy Brief. (2019)

Sanner, B.: Some history of shallow geothermal energy use. In: International Geothermal Days, Germany (2001)

Sanner, B., Knoblich, K.: Schlussbericht zum FuE-Vorhaben. Thermische Untergrundspeicherung auf höherem Temperaturniveau: Begleitforschung mit Messprogramm Aquiferspeicher Reichstag. Institut für Angewandte Geowissenschaften der Justus-LiebigUniversität Gießen (2004)

Sanner, B., Karytsas, C., Mendrinos, D., Rybach, L.: Current status of ground source heat pumps and underground thermal energy storage in Europe. Geothermics 32(4-6), 579-588 (2003). https://doi. org/10.1016/S0375-6505(03)00060-9

Sanner, B., Kabus, F., Seibt, P., Bartels, J.: Underground thermal energy storage for the German parliament in Berlin, system concept and operational experiences. In: Proceedings of the World Geothermal Congress, Antalya, Turkey (2005)

Schmidt, T., Müller-Steinhagen, H.: Die solar unterstützte Nahwärmeversorgung mit saisonalem Aquifer-Wärmespeicher in Rostock Ergebnisse nach vier Betriebsjahren. In: 5. Symposium Erdgekoppelte Wärmepumpen, 8. Geothermische Fachtagung, Landau, Germany (2004)

Schüppler, S., Fleuchaus, P., Blum, P.: Techno-economic and environmental analysis of an Aquifer Thermal Energy Storage (ATES) in Germany. Geotherm Energy 7(1), 669 (2019). https://doi.org/10. 1186/s40517-019-0127-6

Seibt, P., Kabus, F.: Aquifer thermal energy storage-Projects implemented in Germany. In: Proceedings Ecostock. 10th International Conference on Thermal Energy Storage, New Jersey, US (2006)

Silverman, J. (Hrsg.): Energy Storage: A Vital Element in Mankind's Quest for Survival and Progress. Elsevier, Amsterdam, S. 598 (1980)

Snijders, A.L.: Lessons from 100 ATES projects: The developments of aquifer storage in the Netherlands. In: Proceedings Terrastock. 8th International Conference on Thermal Energy Storage, Stuttgart, Germany (2000)

Sommer, W.: Modelling and monitoring of Aquifer Thermal Energy Storage. Impacts of soil heterogeneity, thermal interference and bioremediation. Dissertation, Wageningen University (2015)

Stemmle, R.: Aquifer Thermal Energy Storage in Deutschland - Lebenszyklusanalyse und Potentialstudie. Masterarbeit, Karlsruher Institut für Technologie (2020)

Tissen, C., Benz, S., Menberg, K., Bayer, P., Blum, P.: Groundwater temperature anomalies in central Europe. Environ. Res. Lett. (2019). https://doi.org/10.1088/1748-9326/ab4240

Tsang, C.F.: A review of current aquifer thermal energy storage projects. In: Silverman, J. (Hrsg.) Energy Storage: A Vital Element in Mankind's Quest for Survival and Progress, S. 279-293. Elsevier, Amsterdam (1980)

Ueckert, M., Baumann, T.: Hydrochemical aspects of high-temperature aquifer storage in carbonaceous aquifers: Evaluation of a field study. Geotherm. Energy 7(1), 50 (2019). https://doi.org/10.1186/ s40517-019-0120-0 
Umweltbundesamt: Energieverbrauch für fossile und erneuerbare Wärme. UBA (2020). https://www.umweltbundesamt.de/daten/ energie/energieverbrauch-fuer-fossile-erneuerbare-waerme, $\mathrm{Zu}$ gegriffen: 13. Apr. 2020

Umweltbundesamt: Erneuerbare Energien in Zahlen. UBA (2019). https://www.umweltbundesamt.de/themen/klima-energie/erneuer bare-energien/erneuerbare-energien-in-zahlen, Zugegriffen: 13. Apr. 2020

Werner, D., Kley, W.: Problems of heat storage in aquifers. J Hydrol 34(1-2), 35-43 (1977). https://doi.org/10.1016/0022-1694(77)90 061-0

Würdemann, H., Westphal, A., Kleyböcker, A., Miethling-Graff, R., Teitz, S., Kasina, M., Seibt, A., Wolfgramm, M., Eichinger, F., Lerm, S.: Störungen des Betriebs geothermischer Anlagen durch mikrobielle Stoffwechselprozesse und Erfolg von Gegenmaßnahmen. Grundwasser 21(2), 93-106 (2016). https://doi.org/10.1007/ s00767-016-0324-1

Würdemann, H., Westphal, A., Lerm, S., Kleyböcker, A., Teitz, S., Kasina, M., Miethling-Graff, R., Seibt, A., Wolfgramm, M.: Influence of microbial processes on the operational reliability in a geothermal heat store-Results of long-term monitoring at a full scale plant and first studies in a bypass system. Energy Procedia 59, 412-417 (2014). https://doi.org/10.1016/j.egypro.2014.10.396

Hinweis des Verlags Der Verlag bleibt in Hinblick auf geografische Zuordnungen und Gebietsbezeichnungen in veröffentlichten Karten und Institutsadressen neutral. 